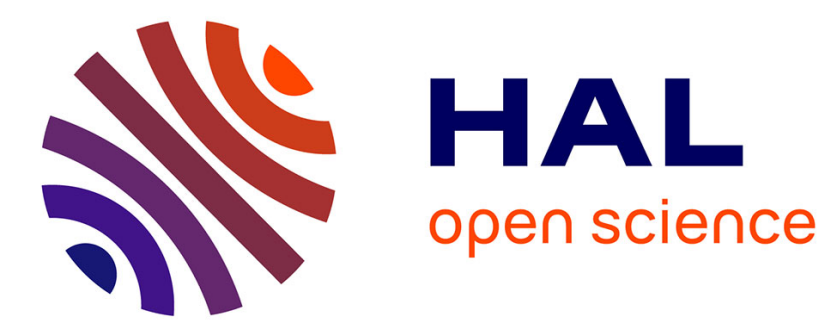

\title{
Modulational instability of dark solitons in three wave resonant interaction
}

\author{
Matteo Conforti, Fabio Baronio, Antonio Degasperis
}

\section{To cite this version:}

Matteo Conforti, Fabio Baronio, Antonio Degasperis. Modulational instability of dark solitons in three wave resonant interaction. Physica D: Nonlinear Phenomena, 2011, 240 (17), pp.1362-1369. 10.1016/j.physd.2011.05.017 . hal-02395368

\section{HAL Id: hal-02395368 https://hal.science/hal-02395368}

Submitted on 5 Dec 2019

HAL is a multi-disciplinary open access archive for the deposit and dissemination of scientific research documents, whether they are published or not. The documents may come from teaching and research institutions in France or abroad, or from public or private research centers.
L'archive ouverte pluridisciplinaire HAL, est destinée au dépôt et à la diffusion de documents scientifiques de niveau recherche, publiés ou non, émanant des établissements d'enseignement et de recherche français ou étrangers, des laboratoires publics ou privés. 


\title{
Modulational instability of dark solitons in three wave resonant interaction
}

\author{
Matteo Conforti ${ }^{a}$, Fabio Baronio $^{a}$, Antonio Degasperis ${ }^{b}$ \\ ${ }^{a}$ Consorzio Interuniversitario per le Scienze Fisiche della Materia, Università di Brescia, \\ Via Branze 38, 25123 Brescia, Italy \\ ${ }^{b}$ Dipartimento di Fisica, and Istituto Nazionale di Fisica Nucleare, Università di Roma \\ "La Sapienza", P.le A. Moro 2, 00185 Roma, Italy
}

\begin{abstract}
We study the propagation of velocity-locked dark triplet solitons in the three wave resonant interaction model. The modulational instability of the plane wave background where the solitons sit prevents the long range propagation. However even a small second order dispersion proves to greatly reduce, or suppress, the modulational instability gain, allowing for effective stable soliton propagation.
\end{abstract}

Keywords: Keywords: Three wave resonant interaction, dark solitons, stability, nonlinear optics

PACS: 05.45.Yv, 02.30.Jr, 42.65.-k

\section{Introduction}

Three-wave resonant interactions (3WRI) are widespread in various branches of physics, as they describe the resonant mixing of waves in weakly nonlinear and dispersive media. The 3WRI model is typically encountered in the description of any conservative nonlinear medium where the nonlinear dynamics can be considered as a perturbation of linear wave propagation, the 
lowest-order nonlinearity is quadratic in the field amplitudes and the phasematching (or resonance) condition is satisfied. Solutions of the 3WRI have been known for a long time $[1,2,3,4,5,6,7]$, and extensive applications are found in nonlinear optics (parametric amplification, frequency conversion, stimulated Raman and Brillouin scattering), plasma physics (laser-plasma interactions, radio frequency heating, plasma instabilities), acoustics (lightacoustic interactions), fluid dynamics (interaction of water waves) and solid state physics $[8,9,10,11,12,13,14,15]$.

Soliton solutions of 3WRI can be classified in three main classes, depending on the boundary conditions. The first one is the class of bright-brightbright (BBB) solitons $[2,6,7,16]$, where the pulses of the three interacting waves vanish sufficiently fast at infinity. The second class, bright-bright-dark (BBD) solitons, is given by two bright pulses which vanish sufficiently fast at infinity and a kink-like pulse which asymptotically behaves like a plane wave $[3,17,18,19,20]$. The third class, dark-dark-dark (DDD) solitons, is given by three locked dark waves [21]. The modulational instability of constant-amplitude backgrounds, i.e. continuous wave (cw) solutions, play an important role for BBD and DDD solitons [22, 23, 24] as it may lead to break-up processes.

The main purpose of this paper is to investigate the propagation and stability of DDD solitons [21] of the 3WRI system. We find that DDD solitons are always unstable because of the modulational instability of the cw background. We also show, however, that the introduction of a quasi-negligible second-order dispersion (or diffraction) is sufficient to drastically reduce the instability gain allowing for effective stable DDD soliton propagation. This 
interesting balance between dispersion and nonlinearity, which implies potential applicability of unstable waves since small dispersion is always present in real contexts, is not new in nonlinear optics, see f.i. the case of the Nonlinear Schroedinger model [25, 26].

Because of computational convenience, we choose to write the 3WRI equations as

$$
\begin{aligned}
u_{1 z}+c_{1} u_{1 y} & =-s_{2} u_{3}^{*} u_{2}, \\
u_{2 z}+c_{2} u_{2 y} & =s_{1} u_{3} u_{1}, \\
u_{3 y} & =\left(c_{2}-c_{1}\right) u_{1}^{*} u_{2},
\end{aligned}
$$

where $u_{1}, u_{2}, u_{3}$ are the wave envelopes, and $z$ and $y$ are, respectively, the transverse and longitudinal coordinates in a spacial process, or, respectively, the space and time coordinates in the time domain. Despite these specifications, we term the parameters $c_{1}$ and $c_{2}$, "velocities", and, with no loss of generality, we assume $0<c_{1}<c_{2}$. The coupling constants $s_{1}, s_{2}$ are just signs: $s_{1}^{2}=s_{2}^{2}=1$. It should be pointed out that this system, being first order, is covariant with respect to arbitrary linear transformations of the $(z, y)$ plane in itself. Therefore, we discuss the system (1) with the understanding that the results we display here can be easily translated into corresponding results for other forms of the 3WRI equations which are covariantly related to it. This observation can be made explicit by displaying the way to transform the general form

$$
\begin{aligned}
& a_{1 t}+v_{1} a_{1 x}=-p_{1} K^{*} a_{2} a_{3}^{*} \\
& a_{2 t}+v_{2} a_{2 x}=p_{2} K a_{1} a_{3}, \\
& a_{3 t}+v_{3} a_{3 x}=-p_{3} K^{*} a_{1}^{*} a_{2}
\end{aligned}
$$


of the 3WRI system as given in [7], into our equations (1). Here $K$ is a complex parameter and $p_{1}, p_{2}, p_{3}$ are signs, $p_{j}^{2}=1$, one of which can be freely chosen ( f.i. $p_{3}$, see below). First note that the labeling of the amplitudes $a_{j}(x, t), j=1,2,3$ is arbitrary because the system (2) is left unchanged by combining transpositions of the indices $1,2,3$ with complex conjugation. Thus one way of fixing the labeling is via ordering the three velocities, for instance $v_{1}>v_{2}>v_{3}$. Next we change coordinates in such a way that the transformed smallest velocity $v_{3}$ vanishes, namely $x=z+v_{3} y, t=y$. Then we observe that, if $K=|K| \exp (i \alpha)$, then the simple transformation $\left(a_{1}, a_{2}, a_{3}\right) \rightarrow\left(a_{1}, a_{2} \exp (i \alpha), a_{3}\right)$ amounts to asking $K$ to be positive real. The final step is setting $p_{1}=s_{2}, p_{2}=s_{1}, p_{3}=-1$ and defining the new wave amplitudes $u_{j}, j=1,2,3$, as

$$
\begin{aligned}
& u_{1}(z, y)=K \sqrt{\frac{c_{2}}{c_{2}-c_{1}}} a_{1}(x, t), \\
& u_{2}(z, y)=K \sqrt{\frac{c_{1}}{c_{2}-c_{1}}} a_{2}(x, t), \\
& u_{3}(z, y)=K \sqrt{c_{1} c_{2}} a_{3}(x, t),
\end{aligned}
$$

with $c_{1}=1 /\left(v_{1}-v_{3}\right), c_{2}=1 /\left(v_{2}-v_{3}\right)$, which turn out to satisfy our system (1), with the condition $0<c_{1}<c_{2}$. Only one crucial point should be kept in mind, namely that in the system (1) the independent variable $y$ should be treated as "evolution" variable. This specification is specially important in investigating the linear stability of solutions.

In the next section 2 we address the modulational stability analysis of the plane wave (cw background) solution of both the (integrable) dispersionless 3WRI system and of its (nonintegrable) dispersive (or diffractive) generalisation, and we show numerical examples of instability gain functions. In section 3 we sketch the main features of the DDD soliton, as described in 
detail in [21], and display numerical evidence of its instability. There we provide also numerical simulations of DDD soliton propagation, without and with the effect of dispersion. Finally the last section 4 presents conclusions and remarks.

\section{Linear stability analysis of $\mathrm{cw}$ solutions}

Before addressing the stability of the DDD soliton which propagates on a background, we investigate in this section the stability of the background itself, namely of the triplet plane wave solution. For future reference, we first generalize the system (1) by adding second-order dispersion terms in the $z$ coordinate:

$$
\begin{aligned}
u_{1 y}+\frac{1}{c_{1}} u_{1 z}+i \beta_{1} u_{1 z z} & =-\frac{s_{2}}{c_{1}} u_{3}^{*} u_{2}, \\
u_{2 y}+\frac{1}{c_{2}} u_{2 z}+i \beta_{2} u_{2 z z} & =\frac{s_{1}}{c_{2}} u_{3} u_{1}, \\
u_{3 y}+i \beta_{3} u_{3 z z} & =\left(c_{2}-c_{1}\right) u_{1}^{*} u_{2},
\end{aligned}
$$

where the real dispersion constants $\beta_{m}(m=1,2,3)$ may be considered small. The reason for this generalization is not only physical, as a small dispersion is always present, but it is also due to the fact that higher order dispersion may reduce (or even suppress) instability [25, 26]. Such effect is found for instance for the Nonlinear Schrödinger equation $i u_{t}+\gamma_{2} u_{x x}+i \gamma_{3} u_{x x x}=\eta|u|^{2} u$ where the stability condition $\eta\left(\gamma_{2}-3 k \gamma_{3}\right)>0$ for the cw solution $u_{c w}(x, t)=$ $A \exp [i(k x-\omega t)]$ implies that, by suitably choosing the wave number $k$ and the higher order dispersion parameter $\gamma_{3}$, stability can be attained even in the self-focusing case $\eta \gamma_{2}<0$. 
In order to proceed in this analysis in full generality, first we construct the family of triplet plane wave solutions of our dispersive 3WRI system (4), namely

$$
u_{j}=A_{j} e^{i\left(k_{j} y-\omega_{j} z\right)} \quad, \quad j=1,2,3
$$

and then we analyze the linear stability of these solutions with respect to small perturbations. Inserting the expressions (5) into the equations (4) yields five relations among the nine plane wave parameters $A_{j}, k_{j}, \omega_{j}, j=$ $1,2,3$, three of them being immediately found, i.e. the resonance conditions $k_{3}=k_{2}-k_{1}$ and $\omega_{3}=\omega_{2}-\omega_{1}$, and the amplitude relation

$$
A_{3}=-i\left[\frac{c_{2}-c_{1}}{k_{3}-\beta_{3} \omega_{3}^{2}}\right] A_{1}^{*} A_{2} .
$$

In order to obtain explicit expressions of the remaining two relations we find it convenient to give a priori (the real value of) $\omega_{1}$ and $\omega_{2}$, together with the complex amplitudes $A_{1}$ and $A_{2}$, and to derive the expressions of the parameters $k_{1}$ and $k_{2}$. This way we first find the implicit relations

$$
\begin{aligned}
& k_{1}=\frac{\omega_{1}}{c_{1}}+\beta_{1} \omega_{1}^{2}-\frac{s_{2}\left(c_{2}-c_{1}\right)}{c_{1}\left(k_{3}-\beta_{3} \omega_{3}^{2}\right)}\left|A_{2}\right|^{2}, \\
& k_{2}=\frac{\omega_{2}}{c_{2}}+\beta_{2} \omega_{2}^{2}-\frac{s_{1}\left(c_{2}-c_{1}\right)}{c_{2}\left(k_{3}-\beta_{3} \omega_{3}^{2}\right)}\left|A_{1}\right|^{2},
\end{aligned}
$$

and then we derive the expression of $k_{3}=k_{2}-k_{1}$ by solving a second degree equation,

$$
\begin{aligned}
& k_{3}=\beta_{3} \omega_{3}^{2}+\frac{1}{2}\left[\frac{\omega_{2}}{c_{2}}-\frac{\omega_{1}}{c_{1}}+\beta_{2} \omega_{2}^{2}-\beta_{1} \omega_{1}^{2}-\beta_{3} \omega_{3}^{2}\right]+ \\
& \frac{1}{2} \sigma \sqrt{\left[\frac{\omega_{2}}{c_{2}}-\frac{\omega_{1}}{c_{1}}+\beta_{2} \omega_{2}^{2}-\beta_{1} \omega_{1}^{2}-\beta_{3} \omega_{3}^{2}\right]^{2}+4\left(c_{2}-c_{1}\right)\left(\frac{s_{2}}{c_{1}}\left|A_{2}\right|^{2}-\frac{s_{1}}{c_{2}}\left|A_{1}\right|^{2}\right)} \\
& \sigma=\operatorname{sign}\left[\frac{\omega_{2}}{c_{2}}-\frac{\omega_{1}}{c_{1}}+\beta_{2} \omega_{2}^{2}-\beta_{1} \omega_{1}^{2}-\beta_{3} \omega_{3}^{2}\right] .
\end{aligned}
$$


This expression completes the construction of the plane wave solutions (5) of the dispersive 3WRI equations (4). We note that, because of the square root in the formula (9), a necessary condition for $k_{1}, k_{2}$ to be real is that the parameters $\omega_{1}, \omega_{2}, A_{1}, A_{2}$ be such that

$$
\left[\frac{\omega_{2}}{c_{2}}-\frac{\omega_{1}}{c_{1}}+\beta_{2} \omega_{2}^{2}-\beta_{1} \omega_{1}^{2}-\beta_{3} \omega_{3}^{2}\right]^{2}+4\left(c_{2}-c_{1}\right)\left(\frac{s_{2}}{c_{1}}\left|A_{2}\right|^{2}-\frac{s_{1}}{c_{2}}\left|A_{1}\right|^{2}\right) \geq 0 .
$$

Now we analyze the stability of these plane wave solutions by considering the following perturbed solutions

$$
u_{j}=A_{j}\left[1+p_{j}(z, y)\right] e^{i\left(k_{j} y-\omega_{j} z\right)}, \quad j=1,2,3 \quad .
$$

By inserting this ansatz into Eqs. (4) and retaining only linear terms in $p_{j}, j=1,2,3$, the three complex perturbations $p_{j}$ obey the linearized equations:

$$
\begin{array}{ll}
p_{1 y}+\left(1 / c_{1}+2 \beta_{1} \omega_{1}\right) p_{1 z}+i \beta_{1} p_{1 z z}+i \mu_{1}\left(p_{1}-p_{2}-p_{3}^{*}\right) & =0, \\
p_{2 y}+\left(1 / c_{2}+2 \beta_{2} \omega_{2}\right) p_{2 z}+i \beta_{2} p_{2 z z}+i \mu_{2}\left(p_{2}-p_{1}-p_{3}\right) & =0, \\
p_{3 y}+2 \beta_{3} \omega_{3} p_{3 z}+i \beta_{3} p_{3 z z}+i \mu_{3}\left(p_{3}-p_{2}-p_{1}^{*}\right) & =0 .
\end{array}
$$

Here we have conveniently introduced the notation

$$
\mu_{1}=-\frac{s_{2}}{c_{1} \mu_{3}}\left(c_{2}-c_{1}\right)\left|A_{2}\right|^{2} \quad, \quad \mu_{2}=-\frac{s_{1}}{c_{2} \mu_{3}}\left(c_{2}-c_{1}\right)\left|A_{1}\right|^{2} \quad, \quad \mu_{3}=k_{3}-\beta_{3} \omega_{3}^{2}
$$

As in the standard Fourier analysis, we consider a periodic perturbation as sum of two Stokes-anti-Stokes sidebands, $p_{m}(z, y)=p_{m}^{(+)} \exp [i(\nu z-\lambda y)]+$ $p_{m}^{(-)} \exp \left[-i\left(\nu z-\lambda^{*} y\right)\right], m=1,2,3$, which reduce the differential equations (12) to a linear $6 \times 6$ homogeneous system of algebraic equations whose 
solvability requires that the (possibly complex) parameter $\lambda$ be one of the six roots of the polynomial

$$
\begin{aligned}
P(\lambda)= & \left(f_{1}^{2}-g_{1}^{2}\right)\left(f_{2}^{2}-g_{2}^{2}\right)\left(f_{3}^{2}-g_{3}^{2}\right)+2 \mu_{1} g_{1}\left(f_{2}^{2}-g_{2}^{2}\right)\left(f_{3}^{2}-g_{3}^{2}\right)+2 \mu_{2} g_{2}\left(f_{1}^{2}-g_{1}^{2}\right)\left(f_{3}^{2}-g_{3}^{2}\right) \\
& +2 \mu_{3} g_{3}\left(f_{1}^{2}-g_{1}^{2}\right)\left(f_{2}^{2}-g_{2}^{2}\right)-\mu_{1}^{2}\left(f_{2}^{2}-g_{2}^{2}\right)\left(f_{3}^{2}-g_{3}^{2}\right)-\mu_{2}^{2}\left(f_{1}^{2}-g_{1}^{2}\right)\left(f_{3}^{2}-g_{3}^{2}\right) \\
& -\mu_{3}^{2}\left(f_{1}^{2}-g_{1}^{2}\right)\left(f_{2}^{2}-g_{2}^{2}\right)-2 \mu_{1} \mu_{2}\left(f_{1} f_{2}-g_{1} g_{2}\right)\left(f_{3}^{2}-g_{3}^{2}\right)-2 \mu_{2} \mu_{3}\left(f_{2} f_{3}-g_{2} g_{3}\right)\left(f_{1}^{2}-g_{1}^{2}\right) \\
& +2 \mu_{1} \mu_{3}\left(f_{1} f_{3}+g_{1} g_{3}\right)\left(f_{2}^{2}-g_{2}^{2}\right)+4 \mu_{1} \mu_{2} \mu_{3}\left[f_{3}\left(f_{1} g_{2}-g_{1} f_{2}\right)-2 f_{1} f_{2} g_{3}\right] \\
& +4 \mu_{1}^{2} \mu_{2} \mu_{3} g_{2} g_{3}+4 \mu_{1} \mu_{2}^{2} \mu_{3} g_{1} g_{3}+4 \mu_{1} \mu_{2} \mu_{3}^{2} g_{1} g_{2} .
\end{aligned}
$$

This expression is written with the following notation:

$f_{j}=\lambda-\nu\left(1 / c_{j}+2 \beta_{j} \omega_{j}\right), j=1,2, \quad f_{3}=\lambda-2 \nu \beta_{3} \omega_{3}, g_{m}=\beta_{m} \nu^{2} \quad, \quad m=1,2,3$,

and $\mu_{m}$ is defined by (13). For any real value of the variable $\nu$, each of the six roots of the polynomial (14) defines one branch of the "dispersion relation", $\lambda=\lambda(\nu)$. The unstable modes occur at those "frequencies" $\nu$ to which there corresponds at least one zero $\lambda(\nu)$ of the polynomial (14) which is complex with nonvanishing imaginary part. We note that this polynomial (14) has real coefficients and therefore its complex zeros come as complex conjugate pairs. For each $\nu$, the instability gain $G(\nu)$ is defined as the maximum of the absolute values of the imaginary part of the six roots:

$$
G(\nu)=\max _{i=1, \cdots, 6}\left\{\left|\operatorname{Im} \lambda_{i}(\nu)\right|\right\} .
$$

Since in general the roots cannot be explicitly obtained, some approximations which lead to analytical results were used in [23]. However these approximations apply only when the root $\lambda(\nu)$ and the frequency $\nu$ are small. This hypothesis is too stringent for the analysis we want to carry on, thus we will solve the general problem by standard numerical methods. 


\subsection{The case with no dispersion}

Let us consider first the dispersionless case, $\beta_{m}=0$. In this case, by numerically exploring the parameter space, we find that, if the amplitudes $A_{j}$ of the three waves are not all vanishing, $A_{1} A_{2} A_{3} \neq 0$, for any real value of $\omega_{1}$ and $\omega_{2}$ there exists an interval of the frequency $\nu$ for which there is at least one pair of complex conjugated roots $\lambda(\nu), \lambda^{*}(\nu)$, with the implication that these plane wave solutions are always unstable. This fact entails that the simulton family reported in the next section does not contain any stable solution. To gain some insight into the three-wave instability problem we consider first the single cw solution, where a complete analytical solution is attainable. Let us start with the simple plane wave solution $u_{1}=A_{1} \exp \left(i k_{1} y-i \omega_{1} z\right), u_{2}=$ $0, u_{3}=0$ with $c_{1} k_{1}=\omega_{1}$. Inserting the perturbed solution $u_{1}=A_{1} \exp \left(i k_{1} y-\right.$ $\left.i \omega_{1} z\right)\left[1+\epsilon^{2} p_{1}(z, y)\right], u_{2}=\epsilon \exp \left(i k_{1} y-i \omega_{1} z\right) p_{2}(z, y), u_{3}=\epsilon p_{3}(z, y)$ in the 3WRI equations (1), yields, at the first order in $\epsilon$, the following system of two linear equations

$$
p_{2 y}+\frac{1}{c_{2}} p_{2 z}+i \omega_{1}\left(\frac{1}{c_{1}}-\frac{1}{c_{2}}\right) p_{2}=\frac{s_{1} A_{1}}{c_{2}} p_{3}, p_{3 y}=A_{1}^{*}\left(c_{2}-c_{1}\right) p_{2}
$$

which, for the Fourier mode $\exp [i(\lambda y-\nu z)]$, implies a second degree equation for $\lambda(\nu)$ whose roots are

$$
\lambda_{ \pm}(\nu)=\frac{1}{2 c_{2}}\left[\nu-\frac{\omega_{1}}{c_{1}}\left(c_{2}-c_{1}\right) \pm \sqrt{\left[\nu-\frac{\omega_{1}}{c_{1}}\left(c_{2}-c_{1}\right)\right]^{2}-4 s_{1} c_{2}\left(c_{2}-c_{1}\right)\left|A_{1}\right|^{2}}\right.
$$

Therefore, if $s_{1}=-1$, the two roots are real and the plane wave solution is stable. On the contrary, if $s_{1}=1$, the solution is unstable, the instability frequency band being $\nu_{-}<\nu<\nu_{+}$with $\nu_{ \pm}=\left(\omega_{1} / c_{1}\right)\left(c_{2}-c_{1}\right) \pm$ 
$2\left|A_{1}\right| \sqrt{c_{2}\left(c_{2}-c_{1}\right)}$. Moreover the instability gain is

$$
G(\nu)=1 /\left(2 c_{2}\right) \sqrt{4 c_{2}\left(c_{2}-c_{1}\right)\left|A_{1}\right|^{2}-\left[\nu-\omega_{1}\left(c_{2}-c_{1}\right) / c_{1}\right]^{2}},
$$

and the maximum instability gain occurs at $\nu=\nu_{\max }=\left(\omega_{1} / c_{1}\right)\left(c_{2}-c_{1}\right)$ with $G\left(\nu_{\max }\right)=\left|A_{1}\right| \sqrt{\left(c_{2}-c_{1}\right) / c_{2}}$.

Similar calculations can be carried out for the other two single plane wave solutions. In particular we find that the plane wave solution $u_{2}=A_{2} \exp \left(i k_{2} y-\right.$ $\left.i \omega_{2} z\right), u_{1}=0, u_{3}=0$ is stable if $s_{2}=1$, while, if $s_{2}=-1$, this solution is unstable, the instability band being $\nu_{-}<\nu<\nu_{+}$with $\nu_{ \pm}=$ $-\left(\omega_{2} / c_{2}\right)\left(c_{2}-c_{1}\right) \pm 2\left|A_{2}\right| \sqrt{c_{1}\left(c_{2}-c_{1}\right)}$. In this case the instability gain takes the expression

$$
G(\nu)=1 /\left(2 c_{1}\right) \sqrt{4 c_{1}\left(c_{2}-c_{1}\right)\left|A_{2}\right|^{2}-\left[\nu+\omega_{2}\left(c_{2}-c_{1}\right) / c_{2}\right]^{2}},
$$

with its maximum $G\left(\nu_{\max }\right)=\left|A_{2}\right| \sqrt{\left(c_{2}-c_{1}\right) / c_{1}}$ for $\nu=\nu_{\max }=-\left(\omega_{2} / c_{2}\right)\left(c_{2}-\right.$ $\left.c_{1}\right)$. On its turn, the plane wave solution $u_{3}=A_{3} \exp \left(i k_{3} y-i \omega_{3} z\right), u_{1}=$ $0, u_{2}=0$ is stable if $s_{1} s_{2}=1$, while, if $s_{1} s_{2}=-1$, this solution is unstable, the instability band being $\nu_{-}<\nu<\nu_{+}$with $\nu_{ \pm}=\omega_{3} c_{1} /\left(c_{2}-c_{1}\right) \pm$ $2\left|A_{3}\right| \sqrt{c_{1} c_{2}} /\left(c_{2}-c_{1}\right)$. The instability gain is

$$
G(\nu)=1 /\left(2 c_{1} c_{2}\right) \sqrt{4 c_{1} c_{2}\left|A_{3}\right|^{2}-\left[\nu\left(c_{2}-c_{1}\right)-c_{1} \omega_{3}\right]^{2}},
$$

with its maximum $G\left(\nu_{\max }\right)=\left|A_{3}\right| \sqrt{c_{1} c_{2}}$ for $\nu=\nu_{\max }=\omega_{3} c_{1} /\left(c_{2}-c_{1}\right)$. Although the general case with $A_{1} A_{2} A_{3} \neq 0$ is not tractable by analytic means, we find it useful to derive approximate expressions of the zeros $\lambda(\nu)$ of the polynomial $P(\lambda)$ (see (14)) and of the instability frequency band $\nu_{-}<$ $\nu<\nu_{+}$. For instance, if we consider amplitudes close to the values $A_{2}=$ 
$A_{3}=0$, with $s_{1}=1$, we find that the expression

$$
\nu_{ \pm}=c_{2} k_{1}-\omega_{2} \pm \sqrt{c_{2}\left(c_{2}-c_{1}\right)}\left|A_{1}\right|
$$

of the instability band well approximates the numerical results shown in next sections. Similarly we obtain the approximate expressions $\nu_{ \pm}=c_{1} k_{2}-\omega_{1} \pm$ $2 \sqrt{c_{1}\left(c_{2}-c_{1}\right)}\left|A_{2}\right|$, and $\nu_{ \pm}=\left(c_{1} c_{2} k_{3}-c_{2} \omega_{1}+c_{1} \omega_{2}\right) /\left(c_{2}-c_{1}\right) \pm 2\left|A_{3}\right| \sqrt{c_{1} c_{2}} /\left(c_{2}-\right.$ $c_{1}$ ) of the instability bands in the cases of amplitudes close to $A_{1}=A_{3}=0$, with $s_{2}=-1$ and, respectively, to $A_{1}=A_{2}=0$, with $s_{1} s_{2}=-1$.

We summarize these findings according to the signs $s_{1}, s_{2}$, and by borrowing the classifying terminology used for the BBB solutions of the 3WRI model (see [7]), i.e. backscattering, explosive and soliton exchange regimes:

- backscattering $\left(s_{1}=s_{2}=1\right)$ : $A_{1}$ unstable, $A_{2}$ stable, $A_{3}$ stable;

- backscattering $\left(s_{1}=-1, s_{2}=1\right)$ : $A_{1}$ stable, $A_{2}$ stable, $A_{3}$ unstable;

- soliton exchange $\left(s_{1}=-1, s_{2}=-1\right)$ : $A_{1}$ stable, $A_{2}$ unstable, $A_{3}$ stable;

- explosive $\left(s_{1}=1, s_{2}=-1\right)$ : $A_{1}$ unstable, $A_{2}$ unstable, $A_{3}$ unstable.

This scenario shows that, in the dispersionless case $\left(\beta_{1}=\beta_{2}=\beta_{3}=0\right)$, the $\mathrm{cw}$ stationary solutions, see (5) with $A_{1} A_{2} A_{3} \neq 0$, are always unstable: for every combination of signs at least one single $\mathrm{cw}$ is modulationally unstable. As a side remark, we also note that the stability of the $u_{1}$ single plane wave in the soliton exchange case is consistent with the stability property of some recently found BBD-type soliton solutions (boomerons) of the 3WRI equations $[18,19,21]$.

Since our main interest is in the stability of the DDD soliton and in the 
next section we show that DDD solitons do not exist in the soliton exchange configuration $s_{1}=-1, s_{2}=-1$, in the following subsection 2.2 , we limit our attention to backscattering configurations, in particular we consider only the case $s_{1}=s_{2}=1$ since the other case $s_{1}=-s_{2}=-1$ is equivalent and can be similarly treated. In fact in the third (explosive) case $\left(s_{1}=-s_{2}=1\right.$ ), all three single cw solutions are unstable and therefore DDD soliton solutions are expected to develop a singularity in a finite propagation interval.

\subsection{Effects of second-order dispersion}

We first consider the stability properties of the single $\mathrm{cw}$ solutions of the 3WRI system (4) with second order dispersion terms. The aim is to display the effect of dispersion by comparing the following analytical results with those given above. Our discussion here is very sketchy since computations, as in the dispersionless case, are standard. Consider first the single plane wave solution $u_{1}=A_{1} \exp \left(i k_{1} y-i \omega_{1} z\right), u_{2}=0, u_{3}=0$ with $c_{1} k_{1}=\omega_{1}+$ $c_{1} \beta_{1} \omega_{1}^{2}$. This solution is again stable if $s_{1}=-1$, and is stable also if $s_{1}=1$ provided the following two conditions are met (all square roots are meant to be positive):

$$
\left\{\begin{array}{l}
\beta_{2} \neq \beta_{3} \\
-\frac{1}{4}+\frac{\omega_{1} c_{2}}{c_{1}}\left[\beta_{3}\left(c_{2}-c_{1}\right)-c_{2} \beta_{2}\right]+c_{2}^{2} \omega_{1}^{2}\left(\beta_{1} \beta_{3}-\beta_{2} \beta_{3}-\beta_{1} \beta_{2}\right)>2 c_{2}\left|A_{1}\right| \sqrt{c_{2}\left(c_{2}-c_{1}\right)\left(\beta_{2}-\beta_{3}\right)^{2}}
\end{array}\right.
$$

Therefore, by appropriately choosing $\omega_{1}$ and the dispersion coefficients $\beta_{1}, \beta_{2}, \beta_{3}$ so that these conditions are satisfied, the effect of dispersion is that of making stable an unstable solution. As for this result, we deem it helpful to briefly point out few computational steps in the derivation of these two conditions. As in the dispersionless case, we start by inserting the perturbed solution 
$u_{1}=A_{1} \exp \left(i k_{1} y-i \omega_{1} z\right)\left[1+\epsilon^{2} p_{1}(z, y)\right], u_{2}=\epsilon \exp \left(i k_{1} y-i \omega_{1} z\right) p_{2}(z, y), u_{3}=$ $\epsilon p_{3}(z, y)$ in (4) to derive the linear equations (compare with (17))

$p_{2 y}+\left(\frac{1}{c_{2}}+2 \beta_{2} \omega_{1}\right) p_{2 z}+i \beta_{2} p_{2 z z}+i \omega_{1}\left[\frac{1}{c_{1}}-\frac{1}{c_{2}}+\omega_{1}\left(\beta_{1}-\beta_{2}\right)\right] p_{2}=\frac{s_{1} A_{1}}{c_{2}} p_{3} \quad, p_{3 y}+i \beta_{3} p_{3 z z}=A_{1}^{*}\left(c_{2}-c_{1}\right) p_{2}$

which, for the Fourier mode $\exp [i(\lambda y-\nu z)]$, implies that the inequality

$$
\left(\gamma_{2} \nu^{2}+2 \gamma_{1} \nu+\gamma_{0}\right)^{2}>4 s_{1} c_{2}\left(c_{2}-c_{1}\right)\left|A_{1}\right|^{2}
$$

should be satisfied for all real values of $\nu$ in order to guarantee stability. Here the three coefficients $\gamma_{0}, \gamma_{1}, \gamma_{2}$ take the expression $\gamma_{0}=\omega_{1}\left[c_{2}\left(\beta_{2}-\beta_{1}\right)-\left(c_{2}-\right.\right.$ $\left.\left.c_{1}\right) / c_{1}\right], \gamma_{1}=1 / 2+c_{2} \beta_{2} \omega_{1}, \gamma_{2}=c_{2}\left(\beta_{2}-\beta_{3}\right)$. Therefore, if $s_{1}=-1$ this single plane wave is stable. If instead $s_{1}=1$, we first note that that the polynomial $\gamma_{2} \nu^{2}+2 \gamma_{1} \nu+\gamma_{0}$ never vanishes for real $\nu$ if and only if $\gamma_{0} \gamma_{2}-\gamma_{1}^{2}>0$ with the implication that $\gamma_{2} \neq 0$, and this provides the first condition $\beta_{2} \neq \beta_{3}$ in (23). If the condition $\gamma_{0} \gamma_{2}-\gamma_{1}^{2}>0$ is satisfied (as it is necessary for stability), one is left with the conditioin

$$
\min _{\nu \text { real }}\left(\gamma_{2} \nu^{2}+2 \gamma_{1} \nu+\gamma_{0}\right)^{2}=\left(\gamma_{0}-\gamma_{1}^{2} / \gamma_{2}\right)^{2}>4 c_{2}\left(c_{2}-c_{1}\right)\left|A_{1}\right|^{2}
$$

which coincides precisely with the second condition given in (23).

By elaborating (23), we find that sufficient conditions for the existence of modulationally stable cw solution are:

$$
\begin{array}{ll}
k_{1}<k_{-} \text {and } & \beta_{2}>\max \left\{\beta_{2+}, \beta_{2-}\right\} \\
k_{1}>k_{+} \text {and } & \beta_{2}<\min \left\{\beta_{2+}, \beta_{2-}\right\}
\end{array}
$$

where

$$
k_{ \pm}=-\beta_{3} \omega_{1}^{2} \pm \frac{2 \sqrt{s_{1} c_{2}\left(c_{2}-c_{1}\right)\left|A_{1}\right|^{2}}}{c_{2}}
$$




$$
\beta_{2 \pm}=\frac{-1+4 \beta_{3} c_{2}\left(c_{2} k_{1}-\omega_{1} \pm 2 \sqrt{s_{1} c_{2}\left(c_{2}-c_{1}\right)\left|A_{1}\right|^{2}}\right)}{4 c_{2}\left[c_{2}\left(k_{1}+\beta_{3} \omega_{1}^{2}\right) \pm 2 \sqrt{s_{1} c_{2}\left(c_{2}-c_{1}\right)\left|A_{1}\right|^{2}}\right]} .
$$

A similar result holds true for the other two single cw solutions. Precisely, the solution $u_{2}=A_{2} \exp \left(i k_{2} y-i \omega_{2} z\right), u_{1}=0, u_{3}=0$ remains stable if $s_{2}=1$ and becomes stable, if $s_{2}=-1$, if the two conditions

$$
\left\{\begin{array}{l}
\beta_{1} \neq-\beta_{3} \\
-\frac{1}{4}+\frac{\omega_{2} c_{1}}{c_{2}}\left[\beta_{3}\left(c_{2}-c_{1}\right)-c_{1} \beta_{1}\right]+c_{1}^{2} \omega_{2}^{2}\left(\beta_{1} \beta_{3}-\beta_{2} \beta_{3}-\beta_{1} \beta_{2}\right)>2 c_{1}\left|A_{2}\right| \sqrt{c_{1}\left(c_{2}-c_{1}\right)\left(\beta_{1}+\beta_{3}\right)^{2}}
\end{array}\right.
$$

are satisfied. Finally, the solution $u_{3}=A_{3} \exp \left(i k_{3} y-i \omega_{3} z\right), u_{1}=0, u_{2}=0$ remains stable if $s_{1} s_{2}=1$ and becomes stable, if $s_{1} s_{2}=-1$, if the two conditions

$$
\left\{\begin{array}{l}
\beta_{1} \neq \beta_{2} \\
-\frac{1}{4}+\frac{\omega_{3} c_{1} c_{2}}{\left(c_{2}-c_{1}\right)^{2}}\left(c_{2} \beta_{2}-c_{1} \beta_{1}\right)+\omega_{3}^{2}\left(\frac{c_{1} c_{2}}{c_{2}-c_{1}}\right)^{2}\left(\beta_{1} \beta_{3}-\beta_{2} \beta_{3}-\beta_{1} \beta_{2}\right)>2 \frac{c_{1} c_{2}}{\left(c_{2}-c_{1}\right)^{2}}\left|A_{3}\right| \sqrt{c_{1} c_{2}\left(\beta_{2}-\beta_{1}\right)^{2}}
\end{array}\right.
$$

are satisfied. These results show that second-order dispersion can indeed suppress the modulation instability of single cw solutions. As for the triplet cw solutions, the previous stability analysis cannot be carried on analytically. Thus, in the next subsection, we show by numerical examples that the analytical results obtained for single plane waves which prove the stabilizing effect of dispersion, still hold for triple $\mathrm{cw}$ solutions in certain ranges of parameters.

\subsection{Numerical examples}

We fix the set of parameters $s_{1}=s_{2}=1, c_{2}=2 c_{1}=2, A_{1}=1$, $\omega_{1}=-3, \omega_{2}=2.25$. Figure 1 a) reports the instability gain $G(\nu)$ for a single wave solution $u_{1}\left(A_{2}=0, A_{3}=0\right)$ and for a three-waves solution $u_{1}, u_{2}, u_{3}$ 

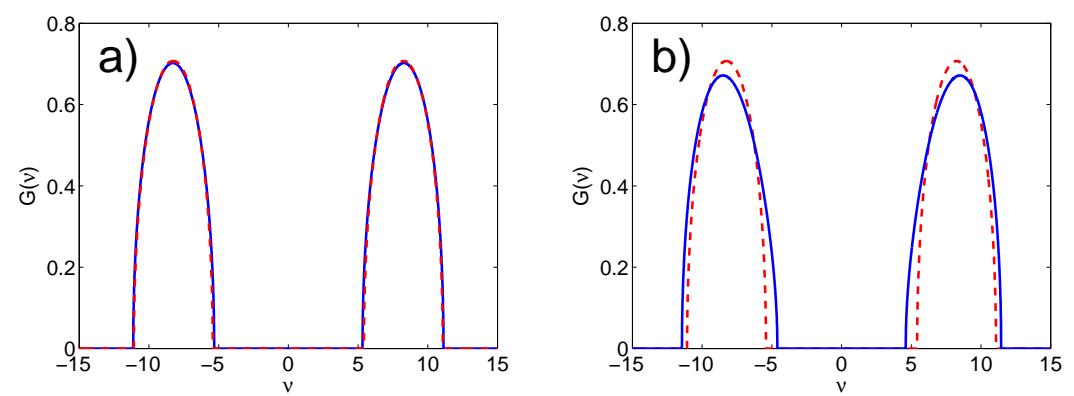

Figure 1: Instability gain for $s_{1}=s_{2}=1, c_{2}=2 c_{1}=2, \omega_{1}=-3, \omega_{2}=2.25, \beta_{m}=0$. a) $A_{1}=1, A_{2}=0$ dashed curve, $A_{1}=1, A_{2}=0.5$ solid curve. b) $A_{1}=1, A_{2}=0$ dashed curve; $A_{1}=1, A_{2}=1.5$ solid curve.

$\left(A_{2}=1 / 2, A_{3}=1 / 8\right)$, in absence of dispersion $\left(\beta_{m}=0\right)$. We can see that the difference between the two instability bands curve is hardly distinguishable. This approximation holds true until the amplitudes of the waves $u_{2}, u_{3}\left(A_{2}\right.$ and $A_{3}$ ) do not exceed the amplitude of the unstable wave $u_{1}$. Figure $1 \mathrm{~b}$ ) shows that the instability bands start differing appreciably in the two cases when $A_{2}=1.5$.

We introduce a small second-order dispersion, in particular we set $\beta_{2} \neq 0$ and $\beta_{1}=\beta_{3}=0$. Considering single stationary $u_{1}$ solutions, from Eqs. (29) and (30) we find that under the conditions $k_{1}<-\sqrt{2}$ and $\beta_{2}>0.0393$ (when $\left.k_{1}=-3\right)$ the instability bands disappear. On the other hand, when all the three $\mathrm{cw}$ waves are present we find numerically that it is not possible to completely eliminate the instability gain; however a drastic reduction of the instability gain is found for values of $\beta_{2}$ higher than the stability threshold of the single wave solution. Figure 2 a) shows that the introduction of a small dispersion leads to the reduction (by an order of magnitude) of the instability gain $G(\nu)$. Moreover Fig. 2 b) shows that for higher amplitude values of the 

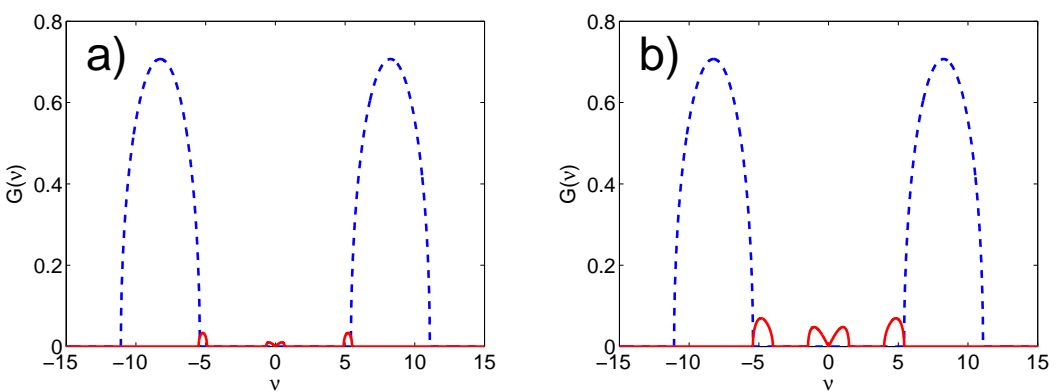

Figure 2: Instability gain for $s_{1}=s_{2}=1, c_{2}=2 c_{1}=2, w_{1}=-3, w_{2}=2.25$. a) $A_{1}=1, A_{2}=0.5$; b) $A_{1}=A_{2}=1$. $\beta_{m}=0$ dashed curve; $\beta_{1}=0, \beta_{2}=0.05, \beta_{3}=0$ solid curve.

waves $u_{2}, u_{3}$, the reduction of instability gain is less pronounced, since we go over the range of the validity of single wave approximation.

We peruse the developmental changes of the instability bands as a function of the dispersion parameter $\beta_{2}$. Figure 3 shows the numerically evaluated instability bands as a function of $\beta_{2}$. Figure 3 a) reports the case of single wave $u_{1}$. The presence of dispersion generates four branches of unstable frequencies, whereas for $\beta_{2}=0$ only two branches exist. The presence of four branches is generated by the folding of the two $\beta_{2}=0$ branches at some critical value of dispersion. This folding completely cancels the unstable bands for large enough $\beta_{2}$.

Figure $3 \mathrm{~b}$ ) reports the case of three-waves cw solution $u_{1}, u_{2}, u_{3}$. No appreciable differences exist from the previous case when $u_{2}, u_{3}$ are small $\left(A_{1}=1, A_{2}=0.5\right)$, in the sense that the unstable branches folding takes place at around the same value of $\beta_{2}$ (see the white line). Only low-gain unstable branches exist for $\beta_{2}>\beta_{2+}$, but they are not discernable in the figure. Increasing $u_{2}, u_{3}$ amplitudes, the effects of the interaction between 

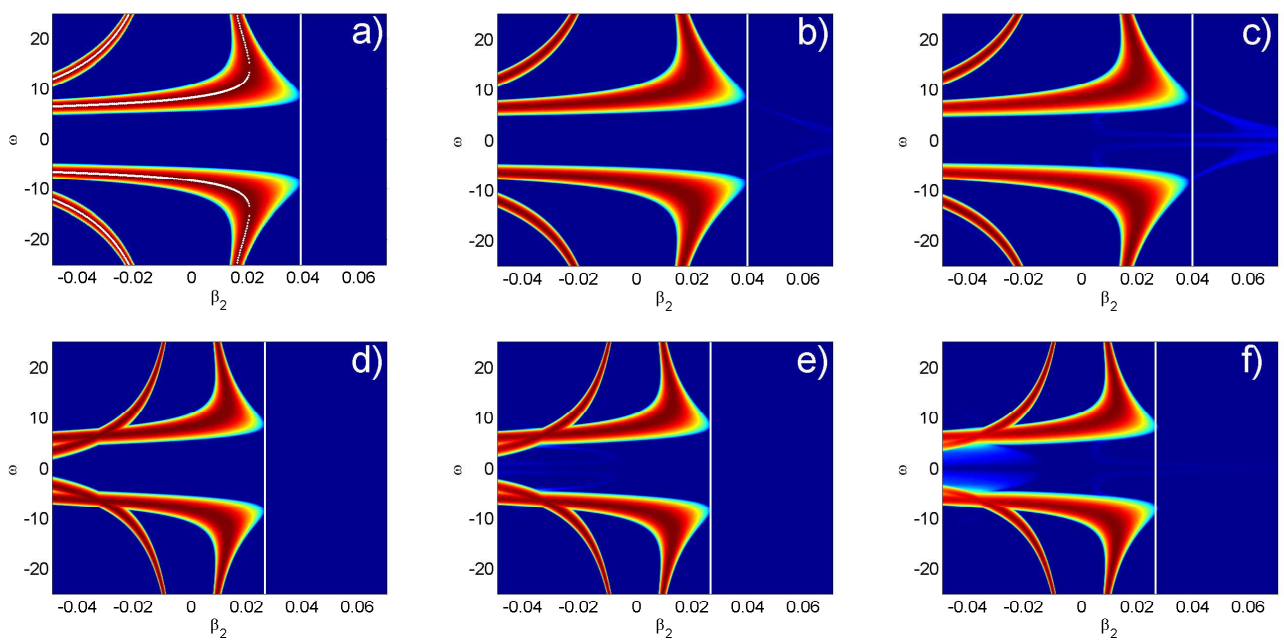

Figure 3: Instability gain. $s_{1}=s_{2}=1, c_{2}=2 c_{1}=2, A_{1}=1, \omega_{1}=-3, \omega_{2}=2.25$. a) $\beta_{1}=\beta_{3}=0, A_{2}=0$. b) $\beta_{1}=\beta_{3}=0, A_{2}=0.5$. c) $\beta_{1}=\beta_{3}=0, A_{2}=1$. d) $\beta_{1}=-\beta_{2}=\beta_{3}, A_{2}=0$. d) $\beta_{1}=-\beta_{2}=\beta_{3}, A_{2}=0.5$. f) $\beta_{1}=-\beta_{2}=\beta_{3}, A_{2}=1$. The vertical white line represents the minimum of $\beta_{2}$ that ensures stability for the single stationary wave $u_{1}$. (

the three waves become more pronounced (Fig. 3 c): additional unstable bands are visible in the $\beta_{2}>\beta_{2+}$ region, which are associated to a non negligible modulational instability gain.

Figures $3 \mathrm{~d}$ )-3 f) show the modulation instability gain considering $\beta_{m} \neq 0$ (i.e., $\beta_{1}=-\beta_{2}=\beta_{3}$ ). The relative sign of the dispersions are chosen to minimize the absolute value of $\beta_{m}$ required to kill the instability bands. The outcomes of the analysis are similar to the previous a)-c) cases, moreover the stabilization can be obtained at lower values of dispersions. 


\section{DDD solitons}

In this section we report the analytical expression of the DDD soliton solution of the 3WRI equations (1) which describes the propagation of three velocity-locked dark pulses.

This expression, which is derived in [21] by a Darboux dressing transformation, reads

$$
\begin{aligned}
u_{j}(z, y) & =A_{j} e^{i\left(k_{j} y-\omega_{j} z\right)}\left(\frac{e^{\xi}+e^{2 i \theta_{j}} e^{-\xi}}{e^{\xi}+e^{-\xi}}\right), j=1,2 \\
u_{3} & =-i\left(\frac{c_{2}-c_{1}}{k_{2}-k_{1}}\right) A_{2} A_{1}^{*} e^{i\left[\left(k_{2}-k_{1}\right) y-\left(\omega_{2}-\omega_{1}\right) z\right]}\left(\frac{e^{\xi}+e^{2 i\left(\theta_{2}-\theta_{1}\right)} e^{-\xi}}{e^{\xi}+e^{-\xi}}\right)
\end{aligned}
$$

where

$$
\xi=p(y-V z)
$$

It clearly describes the propagation of three pulses, all with the same "velocity" $V$, on the background of three (interacting) plane waves. Even if the expression (33) is rather simple, it contains several parameters, whose value has to be computed in a quite involved way which is detailed in [21]. There the spectral characterisation of (33) is given via the standard construction of the one-soliton solution by means of the Darboux dressing transformation with just one simple pole in the complex plane of the spectral variable. For the sake of completeness, we briefly recall the step-by-step procedure to compute this solution :

1. assign the value of the real parameters ("wavenumbers") $k_{1}, k_{2}$, which are arbitrary except for the condition $k_{1} \neq k_{2}$, and of the arbitrary complex amplitudes $A_{1}, A_{2}$. 
2. compute the real "frequencies" $\omega_{1}, \omega_{2}$ by the explicit formulae

$$
\omega_{1}=c_{1} k_{1}+s_{2}\left|A_{2}\right|^{2}\left(\frac{c_{2}-c_{1}}{k_{2}-k_{1}}\right), \omega_{2}=c_{2} k_{2}+s_{1}\left|A_{1}\right|^{2}\left(\frac{c_{2}-c_{1}}{k_{2}-k_{1}}\right) .
$$

3. find the four zeros of the 4-th degree polynomial DIS $(k)$

$$
\operatorname{DIS}(k)=-\frac{4}{27}\left(k_{1}-k_{2}\right)^{2} k^{4}+\Delta_{3} k^{3}+\Delta_{2} k^{2}+\Delta_{1} k+\Delta_{0}
$$

where the real coefficients $\Delta_{n}$ are the following functions of the given parameters $k_{1}, k_{2}, A_{1}, A_{2}$ :

$$
\begin{aligned}
& \Delta_{3}=16\left(k_{1}-k_{2}\right)\left(k_{1}^{2}-k_{2}^{2}+s_{2}\left|A_{2}\right|^{2}-s_{1}\left|A_{1}\right|^{2}\right) \\
& \Delta_{2}=4\left\{\left(s_{1}\left|A_{1}\right|^{2}+s_{2}\left|A_{2}\right|^{2}\right)^{2}+\left(k_{1}-k_{2}\right)^{2}\left[\left(k_{1}+k_{2}\right)^{2}+2 k_{1} k_{2}\right]\right. \\
& \left.\quad-2\left(k_{1}-k_{2}\right)\left[\left(k_{1}-k_{2}\right)\left(s_{1}\left|A_{1}\right|^{2}+s_{2}\left|A_{2}\right|^{2}\right)+3\left(k_{1} s_{1}\left|A_{1}\right|^{2}-k_{2} s_{2}\left|A_{2}\right|^{2}\right)\right]\right\} \\
& \Delta_{1}=2\left\{( k _ { 1 } - k _ { 2 } ) \left[2 k_{1} k_{2}\left(k_{1}^{2}-k_{2}^{2}\right)+9\left(\left|A_{1}\right|^{4}-\left|A_{2}\right|^{4}\right)\right.\right. \\
& \quad+3\left(s_{1}\left|A_{1}\right|^{2}-s_{2}\left|A_{2}\right|^{2}\right)\left(k_{1}^{2}+k_{2}^{2}-4 k_{1} k_{2}\right) \\
& \left.\left.\quad-5\left(s_{1}\left|A_{1}\right|^{2}+s_{2}\left|A_{2}\right|^{2}\right)\left(k_{1}^{2}-k_{2}^{2}\right)\right]+\left(s_{1}\left|A_{1}\right|^{2}+s_{2}\left|A_{2}\right|^{2}\right)^{2}\left(k_{1}+k_{2}\right)\right\} \\
& \Delta_{0}=\left\{k_{1}^{2} k_{2}^{2}\left(k_{1}-k_{2}\right)^{2}-4\left(s_{1}\left|A_{1}\right|^{2}+s_{2}\left|A_{2}\right|^{2}\right)^{3}+8\left(k_{1}-k_{2}\right)\left(k_{2}\left|A_{1}\right|^{4}-k_{1}\left|A_{2}\right|^{4}\right)\right. \\
& \quad+k_{1}^{2}\left|A_{1}\right|^{4}+k_{2}^{2}\left|A_{2}\right|^{4}+18 s_{1} s_{2}\left|A_{1}\right|^{2}\left|A_{2}\right|^{2}\left(k_{1}^{2}+k_{2}^{2}-k_{1} k_{2}\right) \\
& \left.\quad+2\left(k_{1}-k_{2}\right)\left[s_{1} k_{2}\left|A_{1}\right|^{2}\left(2 k_{2}^{2}-k_{1}^{2}-2 k_{1} k_{2}\right)-s_{2} k_{1}\left|A_{2}\right|^{2}\left(2 k_{1}^{2}-k_{2}^{2}-2 k_{1} k_{2}\right)\right]\right\} .
\end{aligned}
$$

If the four zeros of $\operatorname{DIS}(k)$ are all complex there is no soliton solution; the other two possibilities are: two real zeros $k_{-}$and $k_{+}$with $k_{-}<k_{+}$, and four real zeros $k_{-}^{(1)}, k_{+}^{(1)}, k_{-}^{(2)}, k_{+}^{(2)}$ with $k_{-}^{(1)}<k_{+}^{(1)}<k_{-}^{(2)}<k_{+}^{(2)}$.

4. choose the real parameter $\alpha$ in the interval $k_{-}<\alpha<k_{+}$in the case of two real zeros, or in one of the two intervals $k_{-}^{(1)}<\alpha<k_{+}^{(1)}$ or $k_{-}^{(2)}<\alpha<k_{+}^{(2)}$ in the case of four real zeros. Note the difference with respect to the case of the defocusing NLS equation: in the NLS case 
the dark soliton exists for any value of the wave number and amplitude of the background plane wave.

5. compute the complex number $\chi=q+i p$ in the upper half-plane (i.e. with real part $q$ and positive imaginary part $p>0$ ) as solution of the equation

$$
\chi=q+i p=\alpha-\frac{s_{1}}{\alpha+\chi+k_{1}}\left|A_{1}\right|^{2}-\frac{s_{2}}{\alpha+\chi+k_{2}}\left|A_{2}\right|^{2},
$$

which amounts to find the roots of a third degree polynomial. If $\alpha$ has been chosen as at point 4 , these three roots have to be one real and two complex conjugated. Alternatively, $q$ and $p$ can be computed by solving the two coupled real equations

$$
\begin{aligned}
q & =\alpha-\frac{s_{1}\left(\alpha+q+k_{1}\right)}{\left(\alpha+q+k_{1}\right)^{2}+p^{2}}\left|A_{1}\right|^{2}-\frac{s_{2}\left(\alpha+q+k_{2}\right)}{\left(\alpha+q+k_{2}\right)^{2}+p^{2}}\left|A_{2}\right|^{2} \\
1 & =\frac{s_{1}\left|A_{1}\right|^{2}}{\left(\alpha+q+k_{1}\right)^{2}+p^{2}}+\frac{s_{2}\left|A_{2}\right|^{2}}{\left(\alpha+q+k_{2}\right)^{2}+p^{2}}
\end{aligned}
$$

This last equation shows that no solution exists if $s_{1}=s_{2}=-1$.

6. compute the velocity $V$ of the soliton as

$$
V=\frac{s_{1} c_{1}\left|A_{1}\right|^{2}}{\left(\alpha+q+k_{1}\right)^{2}+p^{2}}+\frac{s_{2} c_{2}\left|A_{2}\right|^{2}}{\left(\alpha+q+k_{2}\right)^{2}+p^{2}} .
$$

Note that the assumption $c_{1}<c_{2}$ implies that

$$
\begin{gathered}
c_{1}<V_{-} \leq V \leq V_{+}<c_{2}, \text { if } s_{1}=s_{2}=1, \\
V=c_{1}-\frac{\left(c_{2}-c_{1}\right)\left|A_{2}\right|^{2}}{\left(\alpha+q+k_{2}\right)^{2}+p^{2}},-\infty<V_{-} \leq V \leq V_{+}<c_{1}, \text { if } s_{1}=1, s_{2}=-1, \\
V=c_{2}+\frac{\left(c_{2}-c_{1}\right)\left|A_{1}\right|^{2}}{\left(\alpha+q+k_{1}\right)^{2}+p^{2}}, c_{2}<V_{-} \leq V \leq V_{+}<+\infty, \text { if } s_{1}=-1, s_{2}=1 .
\end{gathered}
$$


No analytic expression of the limit velocities $V_{ \pm}$is known so that their value can be obtained only numerically.

7. compute the asymptotic phase shifts $\theta_{1}$ and $\theta_{2}$ by the formula

$$
\tan \left(\theta_{j}\right)=-\frac{p}{\alpha+q+k_{j}}, j=1,2 .
$$

To summarize this procedure, $s_{1}, s_{2}, c_{1}$ and $c_{2}$ are fixed by the $3 \mathrm{WRI}$ equation (1). Once the free parameters $k_{1}, k_{2}, A_{1}, A_{2}$, which characterize the background plane waves, and the real parameter $\alpha$ are given, the DDD soliton solution is obtained by computing $\omega_{1}, \omega_{2}, q, p, V, \theta_{1}, \theta_{2}$.

Fig. 4 shows a typical analytical DDD soliton solution of the 3WRI.
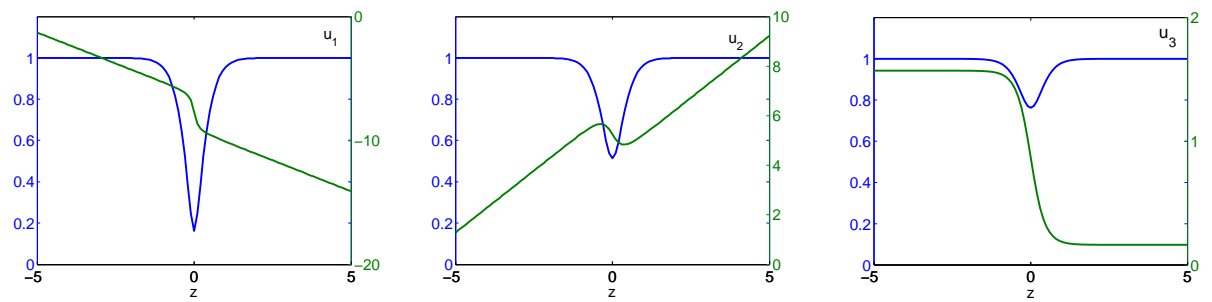

Figure 4: Amplitude and phase of a DDD soliton with parameters $s_{1}=s_{2}=1, c_{2}=$ $2 c_{1}=2, A_{1}=A_{2}=1, k_{1}=2, k_{2}=1, \alpha=-1, V=1.43, p=1.3071, q=-0.7849$, $\theta_{1}=-1.4077, \theta_{2}=1.03$.

As expected from our analysis reported in section 2, Fig. 5 shows the typical numerical DDD soliton propagation in the $z-y$ plane and its instability break-up. Indeed the stable propagation of DDD solitons [21] of the dispersionless $3 \mathrm{WRI}(1)$ is not possible due to the modulation instability of the background components. Similar conclusions are already known and have been investigated in plasma physics $[11,27]$. Nevertheless, we showed that an albeit small second-order dispersion can drastically reduce the mod- 

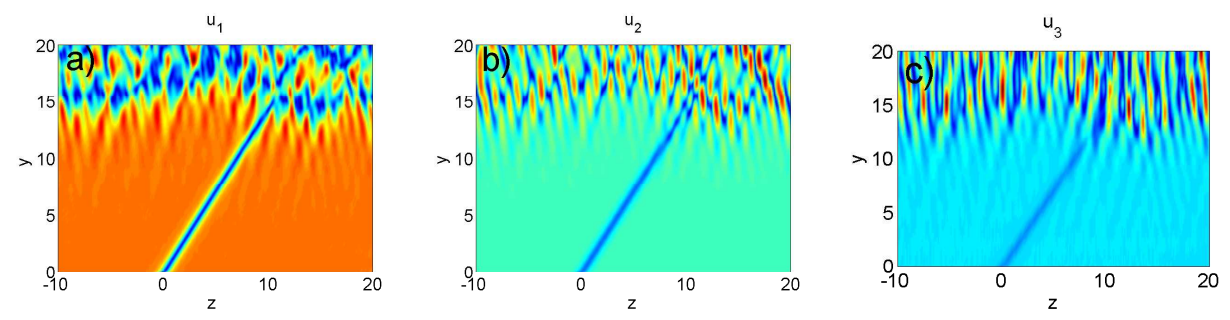

Figure 5: Propagation of the DDD soliton pulses with initial profiles as described in Fig. 4

ulation instability gain of $\mathrm{cw}$ solutions, leading to a quasi-stabilization of the background plane waves.

The introduction of second-order dispersion perturbs the 3WRI system and breaks the 3WRI integrability, preventing the existence, in strict sense, of soliton solutions. Anyway, we consider a small dispersion that perturbs the soliton shape a little upon propagation, but in the meantime it causes a drastic reduction of the instability gain, thus reaching a stable quasi-invariantshape long range propagation.

Figure 6 a) shows the numerical dispersionless propagation of the $u_{1}$ component of a DDD solitons solution in the presence of noise. We consider a quasi-cw background where the DDD soliton sits, in order to mimic real experimental contexts. Figure $6 \mathrm{~b}$ ) reports the power spectrum of the quasi-cw background of wave $u_{1}$ evaluated at $y=8$. It is evident the formation of the unstable band from noise. The instability band location is perfectly captured by the analytical formula (22). On the other hand, Fig. 6 c) shows the numerical propagation of the $u_{1}$ component of the same waves in the presence of a small dispersion $\beta_{1}=-\beta_{2}=\beta_{3}=0.05$. As expected, the presence of dispersion kills the modulation instability and the DDD simulton can 
propagate in a stable fashion. As a matter of fact, the perturbations introduced by second-order dispersion slightly modifies the soliton shape during the propagation: the dark waves undergo little broadening and small ripples are generated.
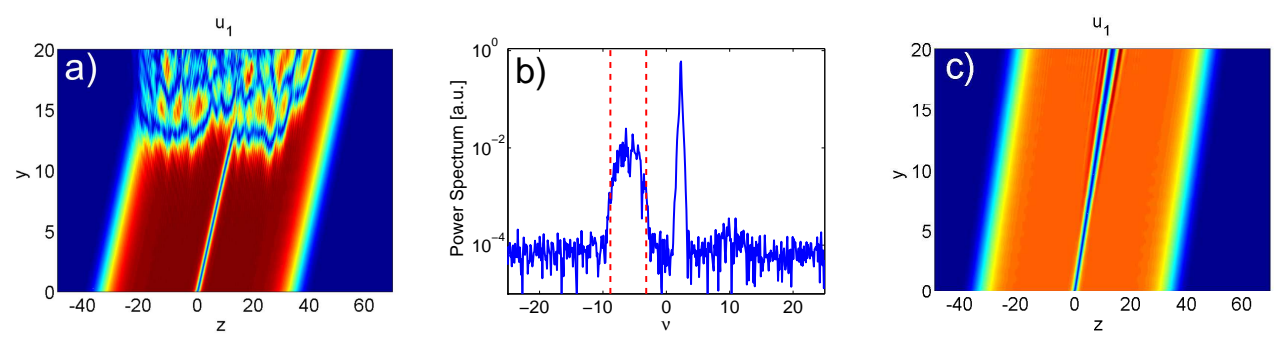

Figure 6: a) Propagation of the $u_{1}$ component of a DDD soliton of the dispersionless 3WRI. Here, $s_{1}=s_{2}=1, c_{2}=2 c_{1}=2$; soliton parameters are $\alpha=1.4, p=0.9536$, $q=0.9615, V=1.04, \theta_{1}=-1.2085, \theta_{2}=-0.3838$. b) Power spectrum of background of wave $u_{1}$ at $y=8$. Dashed lines mark the unstable band calculated from Eq. (22). c) Propagation in presence of dispersion $\beta_{1}=-\beta_{2}=\beta_{3}=0.05$.

\section{Conclusions}

In this paper we have analyzed the stability properties of stationary $\mathrm{cw}$ solutions and dark-dark-dark soliton solutions of the three-wave system. We have found that cw stationary solutions composed of three wave components and dark-dark-dark solitons are always unstable. This could pose severe limitations on applications that tend to exploit long (quasi-cw) pulses in three-wave nonlinear interaction processes. Nevertheless, we have enlightened that the presence of a small dispersion drastically reduce the instability gain, allowing for effective-stable propagation of dark-dark-dark velocity locked nonlinear waves on relevant long distances. 
[1] J. A. Armstrong, S. S. Jha and N. S. Shiren, IEEE J. Quantum Electron. QE-6, 123 (1970).

[2] V. E. Zakharov and S. V. Manakov, JETP Lett. 18, 243 (1973).

[3] K. Nozaki and T. Taniuti, J. Phys. Soc. Jpn. 34, 796 (1973).

[4] Y. Ohsawa and K. Nozaki, J. Phys. Soc. Jpn. 36, 591 (1974).

[5] K. Nozaki, J. Phys. Soc. Jpn. 37, 1124 (1974).

[6] D. J. Kaup, Stud. Appl. Math. 55, 9 (1976).

[7] D. J. Kaup, A. Reiman, and A. Bers, Rev. Mod. Phys. 51, 275 (1979).

[8] E. Ibragimov and A. Struthers, Opt. Lett. 21, 1582 (1996).

[9] M. Conforti, F. Baronio, A. Degasperis, S. Wabnitz, Opt. Express 15, 12246-12251 (2007).

[10] F. Baronio, M. Conforti, A. Degasperis, S. Wabnitz, IEEE J. Quantum Electron. 44, 542-546 (2008).

[11] A. Hasegawa, Plasma Instabilities and Nonlinear Effects (SpringerVerlag, Berlin, 2001).

[12] Y. Tsidulko, V. Malkin, N. Fisch, Phys. Rev. Lett. 88, 235004 (2002).

[13] W. Cheng, Y. Avitzour, Y. Ping, S. Suckewer, N. Fisch, M. Hur, J. Wurtele, Phys. Rev. Lett. 94, 045003 (2005).

[14] A. Craik, Wave Interactions and Fluid Flows (Cambridge Univ. Press, Cambridge, 1985). 
[15] K. Lamb, Geophys. Res. Lett. 34, L18607 (2007).

[16] F. Baronio, M. Conforti, C. De Angelis, S. Wabnitz, A. Degasperis, M. Andreana, V. Couderc, A. Barthelemy, Opt. Express 17, 12889 (2009).

[17] F. Calogero and A. Degasperis, Physica (Amsterdam) 200D, 242 (2005).

[18] A. Degasperis, M. Conforti, F. Baronio and S. Wabnitz, Phys. Rev. Lett. 97, 093901 (2006).

[19] M. Conforti, F. Baronio, A. Degasperis, and S. Wabnitz, Phys. Rev. E 74, 065602(R) (2006).

[20] F. Baronio, M. Conforti, C. De Angelis, A. Degasperis, M. Andreana, V. Couderc, A. Barthelemy, Phys. Rev. Lett. 104, 113902 (2010).

[21] A. Degasperis and S. Lombardo, J. Phys. A: Math. Theor. 42, 385206 (2009).

[22] S. Trillo ans P. Ferro, Opt. Lett. 20, 438 (1995).

[23] Z. H. Muslimani and B. A. Malomed, Physica D 123, 235 (1998).

[24] V. E. Zakharov and L.A. Ostrovsky, Physica D 238, 540 (2009).

[25] A.G. Shagalov, Phys. Lett. A 239, 41-45 (1998).

[26] Woo-Pyo Hong, Opt. Commun. 213, 173-182 (2002).

[27] T. J. M. Boyd and J. J. Sanderson, The Physics of Plasmas, Cambridge University Press, 2003. 